\title{
Influence of Supervisees' Working Alliance on Supervision Outcomes: A Study in Malaysia Context
}

\author{
Nor Mazlina Ghazali, Wan Marzuki Wan Jaafar, Rohani Ahmad Tarmizi, and Sidek Mohd Noah
}

\begin{abstract}
Supervisory working alliance is an essential element in the supervision process. The experiences of supervisees during dyad or supervision process with their supervisors contribute to the levels of relationships (positive or negative) between supervisors and supervisees in their working alliance. The purposes of the research are to determine the relationships between supervisees' working alliance and supervision outcomes and to investigate the influence of supervisees' working alliance on the supervision outcomes. This quantitative study consists of two types of respondents: 1) supervisee (counselor trainee) and 2) supervisor (academic lecturer). A total of 120 supervisees and 18 supervisors participated in the research. This study found that there was a significant relationship between supervisees' working alliance and supervision outcomes (supervisees' satisfaction and performance) $[F(1,116)=49.5,(\beta=1.04, p<0.05), r=.55$ (adjusted $R^{2}=.293$ )]. As a conclusion, the supervisees' working alliance has a unique contribution on the supervision outcomes (supervisees' satisfaction and performance) among supervisees (counselor trainees) in Malaysia.
\end{abstract}

Index Terms-Performance, satisfaction, supervisees' working alliance, supervision outcomes.

\section{INTRODUCTION}

Working alliance in supervision is a main factor that contributes to the positive outcomes. In this context, the supervisees' working alliance can be elaborated as collaborations between goal, task, and emotional bonding between supervisor and supervisee [1]. This is related to supervisees' working alliance which is the independent variable in the research whereas the dependent variable is supervision outcomes. The supervisees' working alliance can be defined as the experienced of connection between supervisor and supervisee [2]. There is a notion about the working alliance as a guiding process to encourage learning amongst supervisees. Supervisees utilize the relationship with the supervisor in order to develop and grow. Working

Manuscript received August 4, 2014; revised October 12, 2014. This work was from $\mathrm{PhD}$ dissertation. This work is supported by the Education Ministry of Malaysia, University Malaysia Sarawak and University of Putra Malaysia. This title of this paper is Influence of supervisees' working alliance on supervision outcomes amongst counselor trainees: A study in Malaysia context.

Nor Mazlina Ghazali is with the University of Putra Malaysia, 43400 Serdang, Selangor, Malaysia (e-mail: gnmazlina@ gmail.com).

Wan Marzuki Wan Jaafar and Sidek Mohd Noah are with the Department of Counseling Education and Counseling Psychology, University of Putra Malaysia, 43400 Serdang, Selangor, Malaysia (e-mail: wanmarzuki@upm.edu.my, smn@upm.edu.my).

Rohani Ahmad Tarmizi is with the Department of Educational Sciences and Technical, University of Putra Malaysia, 43400 Serdang, Selangor, and attached under Institute of Mathematical Research, University of Putra Malaysia, Malaysia (e-mail: rht@upm.edu.my). alliance in supervision is also identified as a relationship between counselor trainee and supervisor that involves social influence [3]-[6]. The supervisory working alliance is a stimulating factor for the counselor trainees or supervisees to transform and supervision process should rely on the mutual agreement on goals, tasks, and emotional bonding. Discussion on the quality of working alliance in supervision is often related to a good relationship between supervisor and supervisee as well as the experience of supervisee along the supervision process [7]. The quality of supervisory working alliance is ranging from positive and negative which represent high (positive) and low quality (negative). Research has reported that a strong relationship between supervisors and supervisees is reflection of a high quality of supervisory working alliance and vice versa.

Supervision outcomes can be defined as the composite of supervisor external events, supervisor characteristics, supervisee characteristics of supervision, and supervisee external events during supervision process [8]. In this particular research, the supervision outcomes are categorized as supervisees' satisfaction and performance. The supervisees' satisfaction can be defined as response from supervisee or counselor trainee towards supervisor's evaluation on qualities and performances, personal behavior along supervision process, and supervisee's level of confidence in delivering their thoughts in supervision [9]. The research also discussed the supervisees' performance as outcomes from the supervision process. The supervisees' performance can be defined as the ability of supervisor in conducting evaluation on supervisee or counselor trainee during supervision process [10]. Supervision performance can be defined as an evaluation on supervisees' clinical process and professional development of the supervisees [2]. Generally, the supervisee's satisfaction and performance can be described as the perceptions of supervisee towards the quality of experiences gained from the supervision process that relates to his or her motivational and practical needs [11].

\section{RESEARCH METHODOLOGY}

\section{A. Initial Stage}

The research utilized a correlational research design that involves supervisees or counselor trainees and supervisors in public universities. Correlational research tests for statistical relationships between variables which involves observation on values of two or more variables and examining the correlation on variables. The researcher hypothesized that there might be a relationship between two variables in this study. Both variables for each of a large number of cases 